\title{
Mechanical circulatory support in the new era: an overview
}

\author{
Kiran Shekar ${ }^{1,2^{*}}$, Shaun D. Gregory ${ }^{1,2}$ and John F. Fraser ${ }^{1,2}$
}

\begin{abstract}
This article is one of ten reviews selected from the Annual Update in Intensive Care and Emergency medicine 2016. Other selected articles can be found online at http://www.biomedcentral.com/collections/ annualupdate2016. Further information about the Annual Update in Intensive Care and Emergency Medicine is available from http://www.springer.com/ series/8901.
\end{abstract}

\section{Background}

Maximal medical therapy can no longer be seen as a justifiable end-point for refractory circulatory shock, at least in well-resourced health settings. Despite improvements in almost all other areas of cardiac and intensive care medicine, refractory cardiogenic shock, defined as cardiac and circulatory failure resulting in organ hypoperfusion [1], continues to have unacceptably high mortality and morbidity from the resultant multiple organ failure. Whilst primary cardiac pathology remains the leading cause of cardiogenic shock, acute cardiomyopathies secondary to conditions such as sepsis and toxic ingestion are not uncommon [2]. The conventional approach to supporting patients with circulatory shock includes reversal of underlying causes when feasible, mechanical ventilation, pharmacological hemodynamic support with or without intra-aortic balloon counter pulsation, renal replacement and other supportive therapy. Whilst mechanical circulatory support (MCS) has always been an attractive option when conventional approaches fail, technological limitations, suboptimal clinical application of available technology and resource limitations have all conspired against its more widespread use.

\footnotetext{
* Correspondence: kiran.shekar@health.qld.gov.au

${ }^{1}$ The University of Queensland, School of Medicine, 4072 Brisbane, Queensland, Australia

${ }^{2}$ The Prince Charles Hospital, Innovative Cardiovascular Engineering and Technology Laboratory, Critical Care Research Group, 4032 Chermside, Queensland, Australia
}

Recently, there is increasing application of extracorporeal membrane oxygenation (ECMO) technology to provide MCS in an incremental fashion either as peripheral or central venoarterial (VA)-ECMO or as univentricular or biventricular assist devices [3, 4]. The use of ECMO in cardiopulmonary resuscitation (CPR) is also expanding with experienced centers reporting favorable outcomes [5]. Other minimally invasive percutaneous ventricular assist devices (pVADs) have also been used in acute settings. Similarly, the implantable, durable, rotary blood pump-driven VADs have revolutionized the care of patients with chronic heart failure or those with acute heart failure who initially need to be stabilized on temporary MCS, and in whom cardiac recovery does not occur [6]. Although total artificial hearts have been used only sparsely, it is expected that their use will increase with the increasing heart failure population and rapid improvements in technology that are currently occurring [7].

In an appropriately aged critically ill patient with no absolute contraindications who is failing medical therapy for circulatory shock, especially of cardiac origin, temporary MCS strategies can now be effectively utilized as a bridge to decision, to recovery, to long-term support devices (such as VADs or total artificial hearts) and/or heart transplantation in an appropriately resourced setting. Such undertakings are resource-intensive and the risk/benefit profile of these therapies and costs are already becoming more favorable. Improved and refined technological advancements, more appropriate selection of patients and better clinical use of these devices will likewise continue.

Bridging a patient from emergent temporary MCS to long term devices and/or heart transplant is a complex multidisciplinary exercise. The choice of the initial rescue MCS strategy has significant bearing not only on limiting further iatrogenic harm in the acute setting, but also on planning long-term strategies in the absence of myocardial recovery. Most of these applications have a steep learning curve and careful planning of perfusion 
strategies and vascular access in a time-critical situation may be challenging. Developing predetermined institutional pathways is critical to success of such MCS programs. It should be recognized that technology has evolved sufficiently and many MCS strategies are now ready for full clinical utilization, though large multicenter trials are still lacking in many areas. Such resource intensive extraordinary therapies raise several questions in relation to resource utilization, ethics, governance, quality assurance and benchmarking, all of which need to be addressed proactively. Although evidence in this area is difficult to generate, collaboration between global centers, establishment of global registries and clinical and science research networks can facilitate the volume and quality of data needed to further augment the clinician's knowledge of when and where these technologies could and should be used. A case vignette will be used to expand on the possibilities, the problems and the pathologies that can be treated with MCS.

\section{Case vignette}

A 28-year old, previously fit male was admitted to a peripheral intensive care unit (ICU) with cardiogenic shock of uncertain origin. Electrocardiogram (EKG) demonstrated no acute ischemic changes, but there was an increase in plasma cardiac troponin I concentration $(21 \mathrm{ng} / \mathrm{ml})$. He deteriorated rapidly following a run of ventricular arrhythmias that required brief $\mathrm{CPR}$ and electric cardioversion. He was subsequently commenced on inotropes and pressors and was intubated and mechanically ventilated. A transthoracic echo demonstrated an akinetic thick ventricular wall, globally diminished cardiac function with a left ventricular ejection fraction (LVEF) of $5 \%$ with no significant valvular abnormalities. An intra-aortic balloon pump (IABP) was inserted. Over the next $6 \mathrm{~h}$ he developed more sustained runs of ventricular arrhythmias with escalating inotrope requirement and early evidence of hepatic and renal dysfunction. The local ECMO center retrieved the patient safely, after establishing peripheral VA-ECMO support, leaving the IABP in situ. Upon arrival at the ECMO center, loss of pulsatility on arterial waveform suggested loss of aortic valve opening, which was confirmed by echocardiography. High intensity anticoagulation, increased inotropes, afterload reduction with nitrates and higher positive end-expiratory pressure (PEEP) were augmented with amiodarone infusion. A follow-up echocardiography hours later demonstrated a distended left ventricle with evidence of some early thrombus in the ventricle and possibly the aortic root. It was predicted that peripheral VA-ECMO was likely to fail and could lead to central thromboembolic and pulmonary complications. Less invasive options for decompression of the left ventricle, such as an atrial septostomy, were considered but excluded.
Other venting options included percutaneous VADs, such as Impella (Abiomed, Aachen, Germany) or TandemHeart (CardiacAssist, Inc., Pittsburgh, PA, USA), but these were not available.

Based on a presumptive diagnosis of acute myocarditis and potential for recovery, his MCS configuration was changed to a temporary biventricular assist device (BiVAD) configuration using two ECMO circuits and centrifugal pumps (CentriMag, Levitronix LLC, Waltham, MA). A Quadrox D Oxygenator (Maquet, Rastatt, Germany) was included in the right ventricular assist device (RVAD) circuit to facilitate gas exchange and temperature control in the early postoperative period. The surgical cannulation strategy employed (transfemoral right atrial [RA] drainage $\rightarrow$ allograft to pulmonary artery $[\mathrm{PA}]$ return; and left ventricular [LV] apex drainage to aorta return) allowed for awakening, mobilization and exercise in bed on BiVAD support. This was necessary to allow physical conditioning and urgent listing for a heart transplant if cardiac recovery failed to occur. Cardiac tissue at the time of LV apical cannulation demonstrated fulminant giant cell myocarditis raising concerns about cardiac recovery.

After hemostasis had been achieved, the oxygenator was removed from the circuit on postoperative day 2 and a tracheostomy was performed to allow weaning from sedation and ventilation. Over the next 2 weeks, the patient was liberated from mechanical ventilation and the tracheostomy removed, with the patient remaining stable on the BiVAD, with some physical recovery, but no cardiac recovery. He was urgently listed for a heart transplant after confirming eligibility. No organ became available for transplant in the following two weeks whilst on temporary BiVAD. Given the patient had a less favorable blood group and a more ambulatory support strategy was needed to move forward, the temporary LVAD was converted to a left side longterm implantable rotary VAD (HVAD, HeartWare, Framingham, MA). Support from the temporary RVAD was continued due to concerns of RV failure post-LVAD implantation.

The early post-VAD insertion was complicated by bleeding requiring reopening but over the next few days a tracheotomy was again performed and rehabilitation was recommenced. Over the next 10 days, the RVAD was removed and the patient was weaned off ventilation and was eventually discharged from the ICU on LVAD support. He received inotropes for RV support in the ward for another 2 weeks. He was relisted for heart transplant a few weeks later. Three months later he received a heart transplant and was discharged to the ward after 1 week in the ICU and subsequently discharged home after a prolonged rehabilitation.

This case illustrates how a spectrum of MCS strategies was used to successfully bridge a young patient with 
acute heart failure to heart transplantation. Equally this case demonstrates the resources, forward-planning and multidisciplinary inputs that are required to provide such a level of care. Although there are understandable concerns regarding the costs associated with these therapies, they are likely to become more widely used, and with appropriate usage, their costs will drop, as with all new technology.

This article will discuss the bridging options in more detail in the sections below to reflect the advancements in MCS and to reinforce the importance of choosing the 'right perfusion strategy for the right patient at the right time'. The intensivist will be a key contributor to MCS both in terms of patient selection, and in determining and enacting at least the initial percutaneous strategies either pre- or post-retrieval from peripheral centers to an advanced MCS center. This article will focus mainly on principles of various MCS strategies and will refer the readers elsewhere for more information on the technicalities of the devices used. Equally, this article discusses more commonly used devices only and is by no means a comprehensive review of all MCS devices in clinical use or development.

\section{Mechanical circulatory support strategies Intra-aortic balloon pumps}

Despite the controversies around their efficacy in the setting of cardiogenic shock [8], IABPs can be seen as a bridge between conventional medical therapy and MCS. IABPs are more widely available than other MCS systems, lower risk, less invasive, easy to institute and may be a useful first-line MCS option while we await definitive evidence for their use in various clinical settings that may lead to cardiogenic shock. More detailed reviews of IABPs and a summary of evidence can be found elsewhere [9]. However, the IABP remains a useful adjunct and it should be noted that while it may improve native cardiac performance by reducing afterload and myocardial oxygen demand, it cannot partially or completely replace cardiopulmonary function. More advanced MCS options need to be considered and an early referral to a MCS center should be considered in a young patient with presumed reversible acute cardiomyopathy or in whom there are no overt contraindications for heart transplantation in the absence of cardiac recovery. Current data suggest that IABPs may assist aortic valve opening in patients requiring peripheral VA-ECMO and they should not necessarily be removed prior to VA-ECMO support.

\section{Venoarterial ECMO}

There has been a significant uptake of ECMO technology in adults since the $2009 \mathrm{H} 1 \mathrm{~N} 1$ Influenza pandemic. This pandemic not only led to many new ECMO centers but also created greater awareness of the process of
ECMO. Success achieved with venovenous (VV) ECMO during the pandemic with contemporary technology has certainly encouraged clinicians to apply ECMO technology to provide cardiorespiratory support in a variety of clinical settings. Providing tailored temporary MCS to patients with acute refractory cardiac failure using ECMO technology is a rapidly evolving area where intervention may be time-critical and mortality is higher than for isolated respiratory failure $[10,11]$.

The indications listed in the Extracorporeal Life Support Organization (ELSO) guidelines for ECMO for cardiac failure in adults are shown in Table 1 [12]. The use of ECMO in the setting of CPR is discussed elsewhere $[13,14]$. International Society for Heart and Lung Transplantation guidelines [15] for MCS provide evidence-based recommendations for long-term MCS options for patients with cardiac failure. These guidelines strongly recommend consideration of the use of temporary MCS in patients with multiorgan failure, sepsis, or on mechanical ventilation, to allow successful optimization of clinical status and neurologic assessment prior to placement of a long-term MCS device. The severity of non-cardiac organ system failures may be used to identify suitable patients and a sequential organ failure assessment (SOFA) score $>15$ has been considered a contraindication to VV-ECMO [16]; similar criteria may be applicable for VA-ECMO or for the use of an ECMO circuit as a temporary VAD.

The underlying cause of cardiac dysfunction and projected time course of recovery, severity of pulmonary dysfunction and projected time course of recovery, functional reserve of each ventricle, the presence and severity of valvular pathology, risk of arterial access and size of vessels, severity of coagulopathy and risk of sternotomy, planned future surgery such as long term VAD or transplant may all have to be considered prior to finalizing an individualized MCS strategy [3].

For patients with predominant cardiac failure and preserved pulmonary function, several MCS strategies may be considered. Central VA-ECMO has traditionally been applied as a bridge to recovery in patients who fail to wean from cardiopulmonary bypass (CPB) after cardiac

Table 1 Extracorporeal Life Support Organization (ELSO) Recommended Indications for ECMO in Adult Patients with Cardiac Failure [12]

\footnotetext{
- Inadequate tissue perfusion manifested as hypotension and low cardiac output despite adequate intravascular volume.

- Shock persisting despite volume administration, inotropes and vasoconstrictors, and intra-aortic balloon counter-pulsation if appropriate.

Typical causes: Acute myocardial infarction, myocarditis, peripartum cardiomyopathy, decompensated chronic heart failure, post cardiotomy shock.

Septic shock is an indication in some centers.
} 
surgery (Fig. 1a). Central VA-ECMO outside this setting in adults is uncommon. Femoral VA-ECMO (Fig. 1b) is the more commonly used MCS modality in adults requiring urgent cardiac support as it can be initiated rapidly and a sternotomy with its concomitant bleeding is avoided. One of the major limitations of peripheral femoro-femoral VA-ECMO is LV afterload mismatch and inadequate LV decompression. Patients with very low native cardiac output states and severe mitral valve regurgitation are at a greater risk of developing hydrostatic pulmonary edema and further reduction of myocardial oxygenation by the distended LV cavity compressing the intracoronary circulation. Although, some centers use an IABP in conjunction with peripheral VA-ECMO to reduce LV afterload and pulmonary congestion, no definitive data exist to support its routine use. Femoral VA-ECMO is also limited by femoral arterial size and thus cannula size and the requirement for distal limb perfusion. Although use of smaller arterial return cannulae may minimize the need for routine back-flow cannula insertion for distal limb perfusion, early insertion of these cannulae should be considered in all these cases until more supportive evidence becomes available.

LV and aortic root stasis from lack of cardiac ejection and failure of aortic valve opening may result in catastrophic intra-cardiac and aortic root thrombosis. Increased intensity of anticoagulation to minimize this risk may precipitate bleeding. Less invasive strategies, such as percutaneous trans-septal left atrial decompression [17] and subxiphoid surgical approaches to drain the left ventricle [18], have been described to reduce LV distension. The residual atrial defect may require surgical correction once the patient has been weaned from the MCS. Use of a pVAD to decompress the distended left ventricle has also been reported in this setting [19] alleviating the need for a high risk septostomy or surgical venting.

Given its less invasive nature (compared to central MCS strategies) peripheral VA-ECMO, with attention to optimal LV afterload, minimizing LV distension with optimal fluid and inotrope therapy, anticoagulation and pulmonary management remains a viable first-line option for patients with isolated acute cardiac failure refractory to conventional management.

\section{Other temporary mechanical circulatory support configurations \\ Configurations based on the ECMO circuit}

The limitations of peripheral VA-ECMO have prompted the use of ECMO devices [20] to facilitate ventricular unloading by changing to a temporary left or right VAD or a BiVAD configuration. Any perfusion strategy that creates a right-to-left shunt requires an oxygenator in the circuit. Oxygenators may additionally facilitate temperature management. This strategy effectively provides biventricular
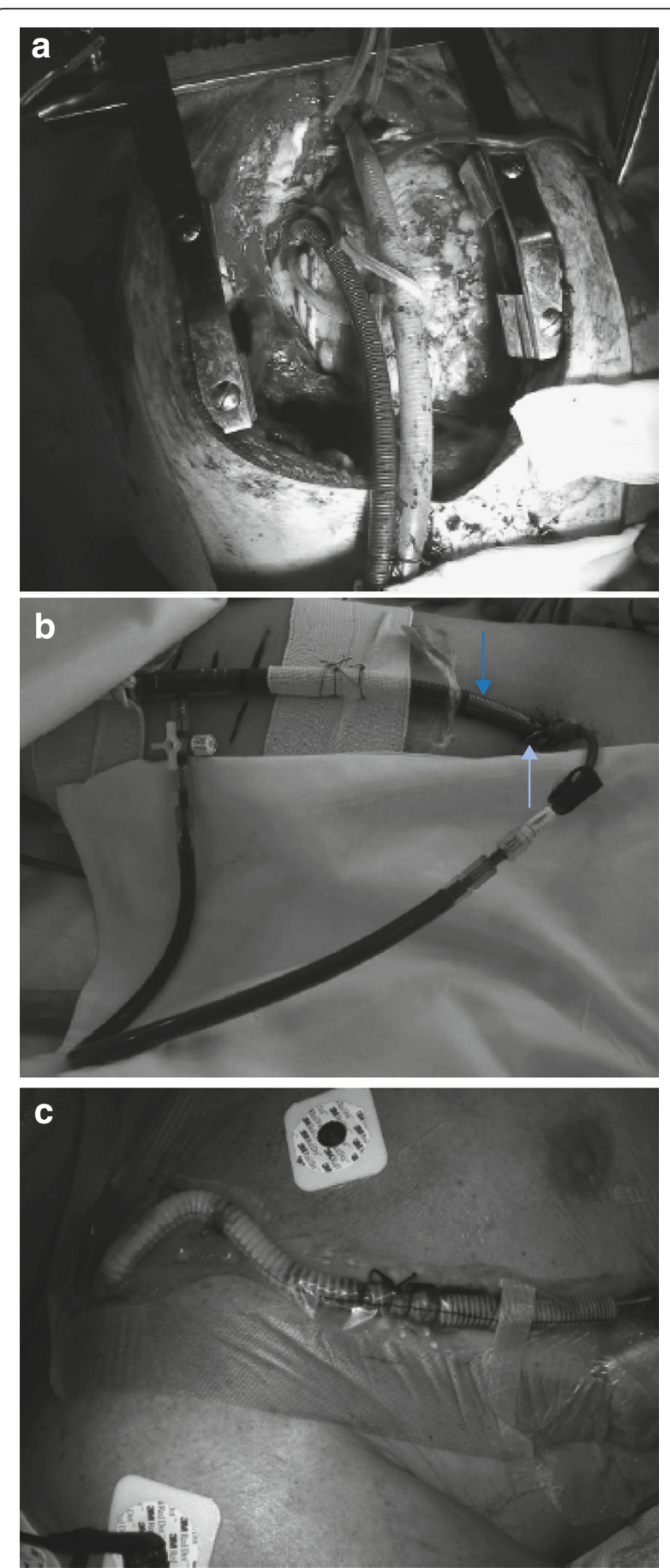

Fig. 1 Cannulation sites for venoarterial extracorporeal membrane oxygenation (VA-ECMO). VA-ECMO can be instituted (a) centrally by cannulating the right atrium/inferior vena cava and the aorta, or peripherally using (b) femoral vein and femoral artery (dark blue arrow arterial return cannula, light blue arrow back flow cannula for distal limb perfusion), or (c) axillary/subclavian artery. The choice is often guided by the clinical setting, expected duration of support and pulmonary function. From [3] with permission 
support and gas exchange through a double (Fig. 2a) or single (Fig. 2b) pump configuration with the ability to cease RV support when not required and thereafter to discontinue the oxygenator. However, this configuration requires a sternotomy and cannulation of the left ventricle (or left atrium), aorta and/or pulmonary arteries. A reoperation (sternotomy or thoracotomy) is then required for explantation of the cannula from the left ventricle or left atrium upon cardiac recovery or for implantation of a long-term mechanical assist device.

RV support for up to several months can be provided with a CentriMag ECMO system through percutaneous femoral venous access to the right atrium and return to the pulmonary artery via a cannulated exteriorized Dacron graft. Alternatively, venous drainage can also be achieved through a centrally placed right atrial cannula. This strategy is described for temporary support of the right ventricle with insertion of a long term LVAD but is applicable to other causes of severe isolated RV dysfunction, such as post-massive pulmonary embolism. Inclusion of an oxygenator into the circuit at this stage ensures adequate oxygenation, $\mathrm{CO}_{2}$ removal and temperature regulation whilst facilitating protective ventilation. Upon RV recovery, the pulmonary artery graft can be ligated and buried upon decannulation without re-sternotomy.

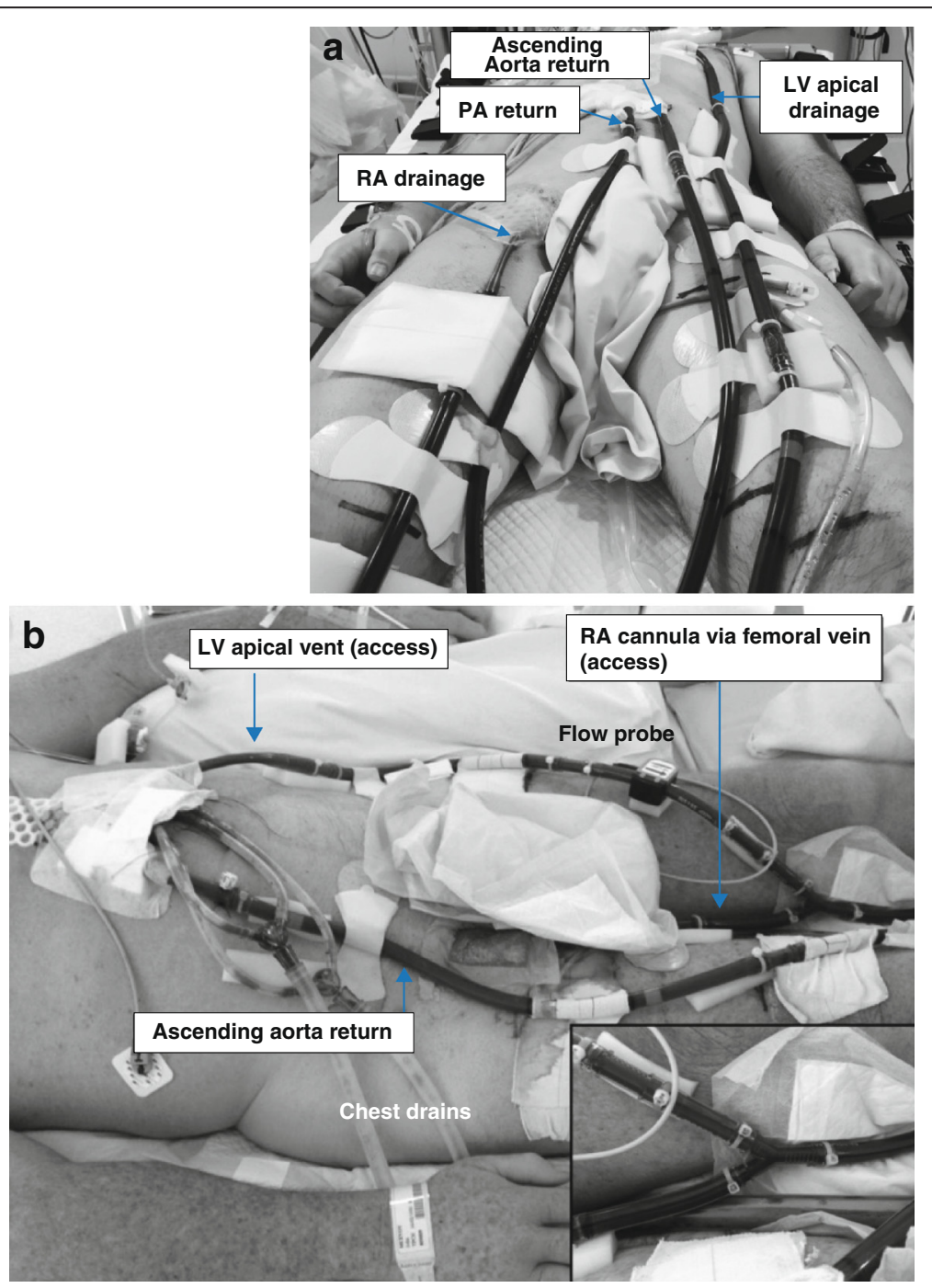

Fig. 2 Temporary biventricular support strategies. a Biventricular assist and oxygenation support using two centrifugal pumps. Cannulation details: transfemoral right atrium (RA) drainage $\rightarrow$ allograft to pulmonary artery (PA) for returning oxygenated blood; and left ventricle (LV) apex drainage to aorta return. An oxygenator was included in the right ventricular assist device circuit. b Biventricular and oxygenation support provided using a single centrifugal pump. Dual drainage cannulas positioned in the LV apically and right atrium transfemorally. Oxygenated blood was returned to the ascending aorta through central cannulation. Insert demonstrates how the two drainage tubes were merged using a Y-connector to enable usage of a single pump 


\section{Percutaneous VADs}

Percutaneously inserted LVADs, such as TandemHeart and Impella [9], are potential options for short-term MCS in the acute setting (Fig. 3). However, there is a paucity of supportive evidence [21] for their use and the complications with arterial access, such as bleeding and limb ischemia, cannot be understated. They may also be

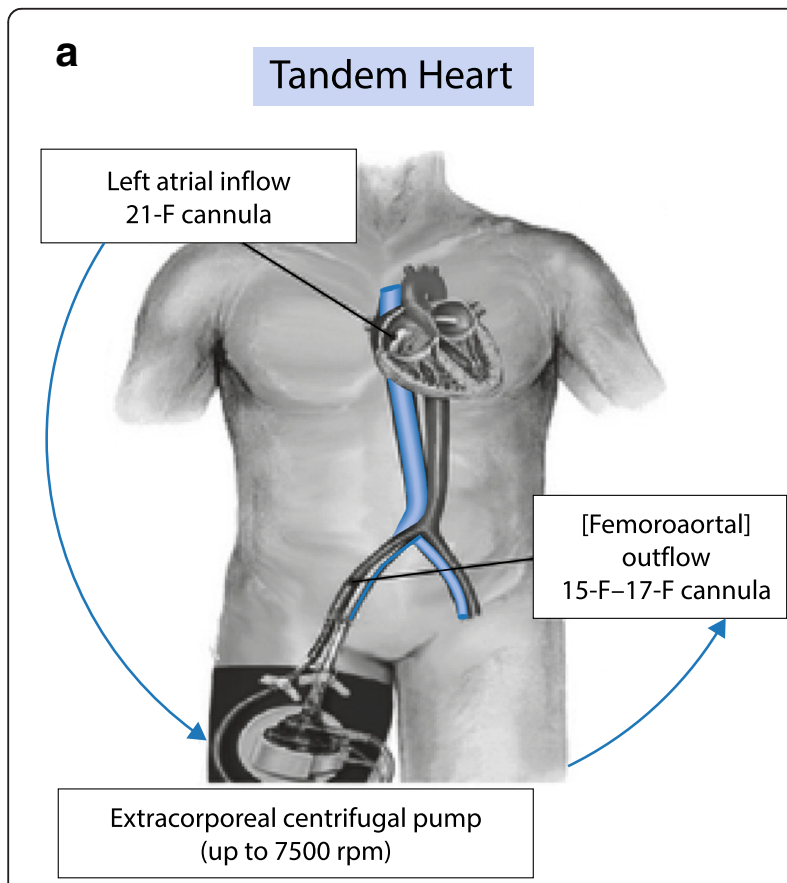

b

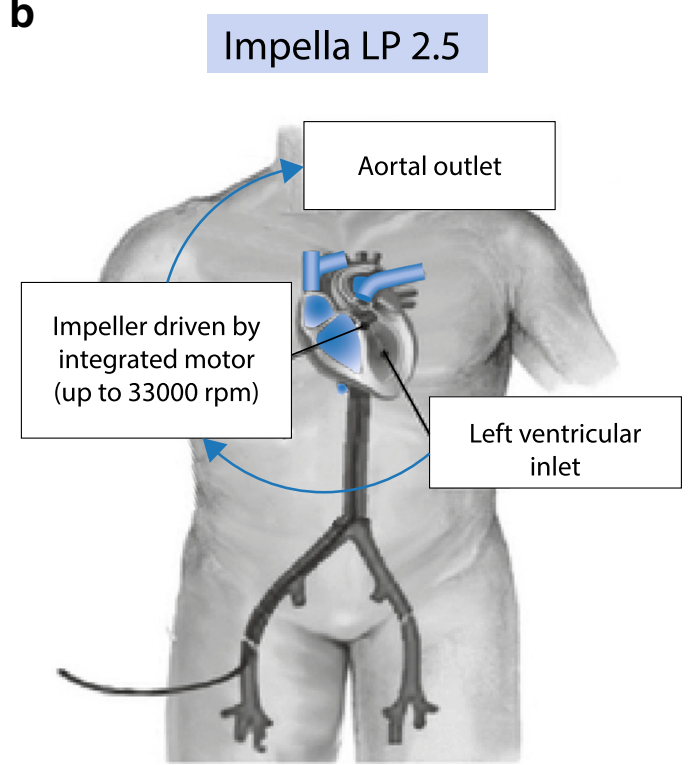

Fig. 3 Schematic representation of two commercially available percutaneous ventricular assist devices. a the TandemHeart pVAD (Cardiac Assist Inc., Pittsburgh, PA, USA), b the Impella pVAD (AbioMed Europe, Aachen, Germany). From [53] with permission viable options to vent the distending left ventricle during peripheral VA-ECMO support. These devices in many ways are likely to form a significant part of our armamentarium whilst providing individualized MCS to a patient with acute cardiac failure.

The TandemHeart uses a centrifugal pump to drain the left atrial blood from a catheter placed transeptally via the femoral vein and returns it to the femoral artery. The Impella uses an axial pump that is inserted retrogradely across the aortic valve via the femoral artery. These devices provide some LV support but lack the ability to provide extracorporeal respiratory support if required. However, there are case reports pertaining to their successful use as RV assist and or biventricular assist devices [22, 23]. Similarly, minimally invasive percutaneous right VADs have been developed (Impella RP system, Abiomed and TandemHeart, CardiacAssist) and may be significant additions to the spectrum of available MCS therapies in the future.

There has been a radical shift in VAD technology and new generation implantable rotary blood pumps are now a viable bridge to destination or heart transplant $[24,25]$. The shortage of appropriate donor organs and the expanding pool of patients waiting for heart transplantation have led to growing interest in alternative strategies, particularly in longer term MCS.

\section{Long-term implantable VADs Indications for support}

Eligible patients with progressive, non-reversible, chronic heart failure may be placed on these devices as bridge to destination or heart transplant. Meticulous patient selection and timely insertion of the device/s is the key to positive outcomes $[6,24]$. The temporary MCS bridging strategies described above in many ways may eliminate the need for placement of these very expensive devices in critically ill patients. This is important, as urgency of VAD placement has also been shown to play a factor in survival. Patients receiving emergent LVADs have a lower rate of survival than patients who are less unwell when the LVAD is implanted [26].

There are several risk models to predict the survival of heart failure patients $[27,28]$. These may be used to identify high-risk patients for potential LVAD therapy. The identified preoperative risk factors for mortality based on the results of the Interagency Registry for Mechanically Assisted Circulatory Support (INTERMACS) indicate that older age, ascites, increased bilirubin, and cardiogenic shock (INTERMACS level 1) are highly associated with post-implant mortality [29]. While it is increasingly obvious that implanting a VAD in these patients is associated with poor survival, refinements in devices and surgical techniques raise an important question: when is it too soon to implant a VAD in a patient 
with progressive, non-reversible, chronic heart failure? The following sections will briefly discuss the available VAD options and common early complications that intensivists may encounter following VAD implantation.

\section{Devices}

Improved results and the increased applicability and durability of LVADs have enhanced this treatment option for end-stage heart failure patients. Results using non-pulsatile continuous flow pumps as a bridge to transplant or destination subsequent to the landmark Randomized Evaluation of Mechanical Assistance for the Treatment of Congestive Heart Failure (REMATCH) Trial [30] are very promising and significantly better when compared with pulsatile LVADs [31]. In 2006, 78 of the 98 implanted devices recorded on the INTERMACS registry were pulsatile, intracorporeal devices [29], whereas in 2013, 2420 of the 2506 implanted devices recorded on the INTERMACS registry were continuous flow intracorporeal devices [29]. Therefore, this section focuses solely on the continuous flow VADs which are commonly used in the clinical setting and does not report on devices no longer clinically available or those under development. A summary of the technical aspects of the devices in this review is provided in Table 2 . We briefly discuss the two commonly used rotary blood pump-based VADs in this article.

The HeartMate II (Thoratec Corporation, Pleasanton, CA) is the most widely implanted rotary blood pump (Fig. 4a), with a second-generation design that relies on a pivot bearing; however minimal wear is reported [32]. To date, over 20,000 HeartMate II devices have been implanted with support duration exceeding 8 years [33].
The HeartMate II received Food and Drug Administration (FDA) approval for bridge to transplant in 2008 and for destination therapy in 2010 [34]. In 2014, Thoratec started clinical trials for the HeartMate 3, a third-generation centrifugal flow design with a magnetically levitated impeller to increase blood flow gaps and reduce blood trauma. The HeartMate 3 includes a small artificial pulse to enhance pump washout and textured blood-contacting surfaces to encourage tissue integration. The HeartWare HVAD (Fig. 4b) is a centrifugal, third generation device with passive magnetic and hydrodynamic forces levitating the impeller and two axial flux motors for redundancy in case one fails. The HVAD has also been used for RV support [35], although CE or FDA approval for this purpose has not been obtained. In 2015, HeartWare started clinical trials of the miniaturized MVAD, an axial flow pump approximately one-third the size of the HVAD with similar impeller levitation principles and capable of less-invasive implantation due to its smaller size [36].

Apart from early postoperative hemostatic complications, a major issue in the early postoperative course is that of RV failure. While controversy remains around pre-emptive mechanical RV support using a temporary RVAD based on ECMO circuitry or an implantable LVAD on the right (there is no customized, long-term rotary RVAD at this stage), it should be noted that re-operation to institute mechanical RV support once RV failure sets in adds to mortality and morbidity in these patients [37]. A high index of suspicion preoperatively and vigilance and prompt escalation of pharmacological and mechanical RV support intra- and postoperatively is the key. We refer the readers elsewhere for a more detailed summary of outcomes and complications [38].

Table 2 Technical summary of clinically available, long-term implantable rotary blood pumps

\begin{tabular}{|c|c|c|c|c|}
\hline Device & Size (mm) & Weight (g) & Speed (RPM) & Flow rates (I/min) \\
\hline \multicolumn{5}{|l|}{ Axial Flow } \\
\hline Thoratec, HeartMate II & $60 \times \phi 40$ & 375 & $6,000-15,000$ & $\geq 10$ \\
\hline Reliant Heart, Heart Assist 5 & $71 \times \phi 30$ & 92 & $7,500-12,500$ & $\geq 10$ \\
\hline Jarvik Inc, Jarvik 2000 & $55 \times \phi 25$ & 85 & $8,000-12,000$ & $\geq 7$ \\
\hline \multicolumn{5}{|l|}{ FlowMaker } \\
\hline Berlin Heart, INCOR & $120 \times \phi 30$ & 200 & $5,000-10,000$ & $\geq 7$ \\
\hline \multicolumn{5}{|l|}{ Centrifugal Flow } \\
\hline HeartWare, HVAD & $57^{\mathrm{a}} \times \phi 50$ & 160 & $1,800-4,000$ & $\geq 10$ \\
\hline Terumo, DuraHeart & $45 \times \phi 72$ & 540 & $1,200-2,600$ & $\geq 10$ \\
\hline Thoratec, HeartMate III & $30 \times \phi 69$ & 474 & $2,000-5,500^{b}$ & $\geq 10$ \\
\hline \multicolumn{5}{|l|}{ Mixed Flow } \\
\hline CircuLite, Synergy & $50 \times \phi 12$ & 25 & $20,000-28,000$ & $\geq 3.5$ \\
\hline HeartWare, MVAD & $50 \times \phi 21$ & 58 & $16,000-28,000$ & $\geq 10$ \\
\hline
\end{tabular}

RPM revolutions per minute, $\phi$ diameter

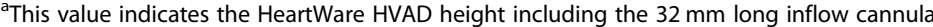

${ }^{\mathrm{b}}$ This value is taken from minimum and maximum pump speeds shown on the pressure head versus volume flow rate curve in [54] 


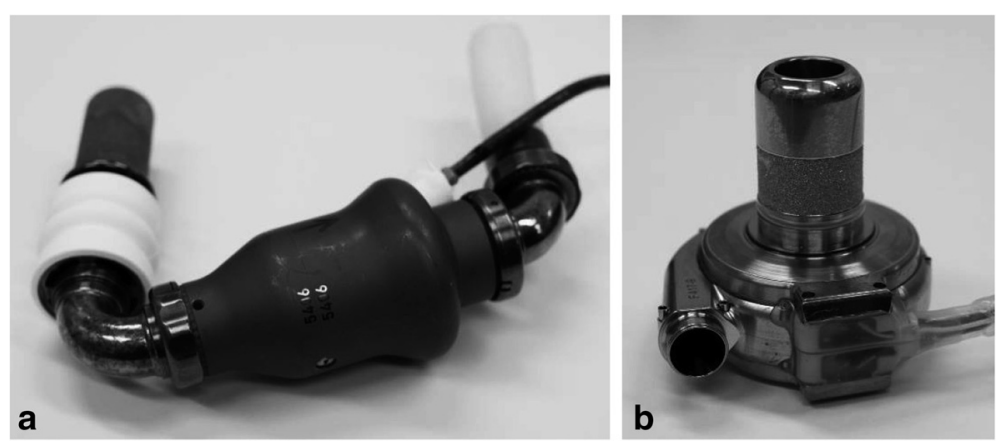

Fig. 4 Two commonly used rotary ventricular assist devices (VADs). a Thoratec HeartMate II, b HeartWare HVAD

\section{Total artificial hearts}

Compared to the dramatic increase in continuous intracorporeal pump implants over the last decade, clinical use of total artificial hearts has been much slower. In 2007, the INTERMACS database reported 22 pulsatile intracorporeal total artificial heart implants, which had increased only to 66 by 2013 [29]. The lack of a longterm, low-wear device with small wearable components, as seen in the latest generation of VADs, may have contributed to the slow uptake of total artificial hearts. Meanwhile, the 'safety net' provided with a VAD, where remnant ventricular contractility may sustain life until emergency intervention, could also explain why total artificial hearts are only used when absolutely necessary.

Although several total artificial hearts, such as the Liotta-Cooley, Akutsu III and the AbioCor devices, have been used to support patients [7], these devices are no longer used clinically. The Carmat (Vélizy Villacoublay, France) total artificial heart is currently in clinical use, however very few patients have been supported since the first implant in December 2013. Meanwhile, the use of a dual LVAD configuration for total artificial heart support has been reported using dual HeartMate II $[39,40]$ or HeartWare HVAD [41] devices; however clinical experience with this technique is limited. The only total artificial heart currently available to fully support the circulation for which there is substantial clinical experience is the SynCardia total artificial heart (SynCardia, Tucson, AZ).

Initially developed as the Jarvik 7 and renamed as the Symbion, Cardiowest and now SynCardia total artificial heart, this pulsatile first generation device consists of two pneumatically operated chambers which provide total systemic and pulmonary flow. A pneumatic driver, for which a $6.1 \mathrm{~kg}$ portable version now exists, supplies pulses of compressed air through percutaneous leads to the left and right chambers to deliver almost $70 \mathrm{ml} /$ beat. The beat rate can be changed to deliver flow rates up to $9.5 \mathrm{l} / \mathrm{min}$ from the device, which weighs
$160 \mathrm{~g}$. Unidirectional flow is achieved with four tilting valves, which have reportedly never failed, while the pumping diaphragms have a failure rate of less than $1 \%$ [42]. Although the SynCardia total artificial heart has been in clinical use for several decades with CE and FDA approval for bridge-to-transplant (1999 and 2004 respectively) and FDA investigational device exemption for destination therapy (2015), widespread clinical use has been slow with over 1440 implants to date [42]. The longest duration of support with the SynCardia total artificial heart currently stands at 1374 days [42]; however typical support duration is closer to 15-90 days at different centers [7]. Meanwhile, SynCardia have recently had FDA investigational device exemption approval for a smaller total artificial heart version with $50 \mathrm{ml}$ pneumatic chambers.

The quest for a durable, safe, practical and affordable total artificial heart continues and rotary blood pump technology has the potential to deliver the same. In the meantime, the temporary and long term MCS options discussed thus far will have to be used in an individualized, tailored fashion so that positive patient outcomes may be achieved whist making the most of available technology.

\section{Quality assurance, governance and benchmarking}

Whilst MCS devices appear to be attractive technologies, the multidisciplinary teamwork required is substantial and one key to success is a smooth decision-making paradigm including all the relevant players, where all the options discussed are considered, but acted upon in a timely fashion, thus delaying any further physiological deterioration of the patient. Currently, clinicians working with MCS must deal with a developing technology still with substantial risks. To optimize outcome in patients requiring $\mathrm{MCS}$, clearly defined work unit guidelines and protocols are needed that can minimize the risks associated with the currently imperfect technology. The risk/benefit ratio of MCS will be improved further if 
the multiple stakeholders in this field collaborate in a silo free research environment - bringing together fields as disparate but inextricably linked as engineering, science, medical, surgical, intensive care and allied health. Governance, quality assurance and benchmarking of MCS practices are also essential to determine optimal team make-up and volume of cases/types of case to be undertaken in each advanced center to maintain adequate skills and knowledge base. Centers that are proficient in the full array of MCS options discussed in this chapter may serve as a 'hub' for several peripheral centers that can initiate timely temporary MCS (typically peripheral VA-ECMO). Case volume and outcome relationships are very likely to exist in such specialized areas of care and maintaining staff training and individual/institutional accreditation also needs to be considered. Similarly, patient referral patterns to these advanced MCS centers from other centers will change, creating pressure on transfer capabilities, intensive care and hospital resources. As the field evolves, data from ELSO, INTERMACS and other local and international registries will allow clinicians to audit and improve upon their clinical practice. Governance and organizational issues must be addressed at a number of levels, and this discussion will require health economists and policy makers to be involved $a b$ initio.

\section{Resource and ethical issues}

The resource intensive nature of MCS therapies is a major barrier for their global uptake. Equally, developing, validating and clinically testing is a resource intense exercise as well. Industry, clinicians, researchers and policy makers will all have to work together in delivering these MCS devices, which can radically change the way we deal with a leading cause of death worldwide, i.e., cardiovascular disease. One of the most important ethical dilemmas faced by clinicians who are so invested in evidence-based medicine is the individual centric nature of MCS, in which 'one size doesn't fit all' and there will be a learning curve where patients will have to be offered these extraordinary therapies (especially the temporary MCS options as bridge to decision/recovery/ device/transplant) outside the comfort zone of compelling favorable evidence from a randomized controlled trial. Equally it may be emotionally challenging for the staff involved to see their patients not get a positive outcome despite spending long periods of time in intensive care/hospital on various bridging MCS options and then not reach their ultimate goal of destination device or heart transplantation. Thus, MCS can raise significant ethical issues [43-45] in a world that is diverse from social, cultural and financial points of view. None of these factors should be a deterrent towards an ultimate goal of delivering temporary and longer term MCS devices.

\section{Research priorities and advancing the field of mechanical circulatory support}

Despite an ongoing debate on the merits and demerits of a non-pulsatile circulation, which results in the setting of many of these MCS strategies based on rotary blood pumps, short-term clinical results are encouraging. Equally, more research is needed in the area of microcirculatory alterations in the presence of a non-pulsatile circulation. When it comes to MCS, the simplicity of rotary blood pumps results in less shear forces on blood cells and biotrauma as compared to displacement pumps.

\section{Temporary acute mechanical circulatory support}

Peripheral VA-ECMO is an imperfect but viable tool in patients with cardiac failure. Improvement in cannula design for ease of insertion eliminating the need for backflow cannulation will improve its risk/benefit ratio. Technological solutions to minimize the afterload increases imposed on the native heart and timed afterload reduction if possible to promote aortic valve opening and minimize LV distension may alleviate the need for invasive central strategies. Development of minimally invasive LV venting strategies is also desirable. Similarly, an advanced understanding of the biological burden of adding an extracorporeal circuit with vast surface area is poorly understood and basic science research to advance our understanding of the pathophysiology of ECMO is the first step towards optimization of hematological, inflammatory, infectious and pharmacokinetic issues that add to the morbidity of ECMO. Clinical research currently must focus on establishing best practice guidelines for use of ECMO in the clinical setting of cardiogenic shock or severe cardiorespiratory failure. Paracorporeal short-term VADs are an attractive but underutilized option for acute MCS and further research should focus on improving the durability of these devices [46], minimizing morbidity especially ischemic limb complications and generating much needed evidence for their use.

\section{Long-term mechanical circulatory support-vads and total artificial hearts}

With durable and miniaturized pumps that have proven clinical success, and the upcoming evolution to even smaller devices, future development of MCS should focus on developing the system around the pump to reduce postoperative complications. The two most frequent adverse events identified in the sixth INTERMACS report [29] were bleeding and infection. Bleeding is partly due to the anticoagulation regime following VAD implantation and acquired von Willebrand syndrome [47, 48]. Research will simultaneously focus on improving implantation techniques, through development of less-invasive procedures off-CPB, whilst developing a more complete understanding 
of the complex blood-VAD interaction. The relative lack of pulsatility seen with rotary VADs is known to at least contribute to gastrointestinal bleeding, arteriovenous malformations, hemolysis and pump thrombosis [49]. Interest in pulsing rotary blood pumps (i.e., speed modulation) has therefore increased and should be further explored, with the potential added benefits of increased aortic valve opening, coronary perfusion, baroreflex sensitivity and ventricular washout.

Early percutaneous driveline infections are a feared and catastrophic complication [50], while late-onset driveline infections are the equal highest cause of death in VAD patients after 4 years of support [29]. Therefore, improved driveline development and implantation techniques along with clinically approved transcutaneous energy transfer systems are required. Meanwhile, right heart failure is a frequent and potentially fatal complication following LVAD implantation $[51,26]$ and is associated with worse outcomes [29]. Further research and clinical experience with dual LVAD biventricular support configurations must be carried out, while the continued development of RVAD-specific devices and rotary total artificial heart technology should be completed in parallel to source an optimal solution. The low preload and high afterload sensitivities of these rotary VADs can result in venous congestion and ventricular suction events [52]. Physiological control systems, which actively change pump speed based on hemodynamic feedback variables, have been developed but not clinically accepted. Further research should focus on progression into clinical practice; however, this may depend on the development of a reliable, implantable sensor (pressure and/or flow) for hemodynamic feedback.

\section{Global databases and registries}

The diversity of MCS techniques, especially in the acute setting, adds to the challenges of performing meaningful clinical studies in the area. Preliminary understanding of global MCS practices and inherent heterogeneity in practice is a key pre-requisite. Similar to INTERMACS, establishment of a broader acute MCS registry collecting data in the acute setting using ECMO or pVAD based strategies may be an important step forward. The ELSO registry, over time, has become an invaluable tool; however, current data collection does not involve advanced temporary MCS strategies discussed in this article and some modifications to reporting structure may have to be made to ensure that all MCS runs based on an ECMO circuit are reported. It should be noted that most reporting to these global databases remains optional.

\section{Global trial networks}

Without global engagement, it will be impossible to drive the high quality randomized trials necessary for the safe development of these therapies. In the last few years, ELSO has developed strong regional representations with most geographical regions having their own active chapters that promote training, education and research. Networks such as the International ECMO Network (ECMONet), and the International Society for Rotary Blood Pumps have been formed to foster further research and development of best practice guidelines in the field of MCS. These existing platforms can be effectively used to design and conduct high quality MCS trials. Responsible and ethically-sound industry engagement is also paramount when designing such trials.

\section{Conclusion}

This review highlights the spectrum of available therapies for acute and chronic heart failure patients and circulatory shock in general. The flexible nature of MCS configurations allows for an individualized approach driven by the ultimate goal of achieving organ recovery or bridging to long-term options. Intensivists, when faced with severe cardiac failure, especially in younger patients, may have to consider MCS as a viable option and initiate timely referrals. Similarly in patients with chronic heart failure, timely insertion of long-term VADs prior to development of irreversible end-organ dysfunction will minimize postoperative morbidity and eliminate the need for short-term temporary bridging mechanical support. Some patients will need short-term mechanical right heart support with a temporary RVAD and in others a long-term BiVAD may be inevitable and customized implantable RVADs may become a reality in future. Both VAD and total artificial heart technology will see many more refinements with time and it is difficult to predict which of these devices will prove to be a perfect bridge to heart transplant or destination. Whilst these technologies may seem excessively complex and expensive, this was once said of dialysis. In the current era, in an appropriately resourced setting, no eligible patient should die on maximal medical therapy without MCS being considered.

\section{Competing interests \\ The authors declare that they have no competing interests.}

\section{Authors' contributions}

KS wrote the initial draft manuscript. SD and JF wrote sections of the manuscript, reviewed and edited the manuscript. All authors read and approved the final manuscript.

\section{Acknowledgements}

Ms Lynette Munck for administrative support and proof reading the manuscript. John Fraser currently holds a Health Research Fellowship awarded by the Office of Health and Medical Research, Queensland, Australia. The authors would like to recognize the financial assistance provided by the National Health and Medical Research Council (APP1079421). The contents are solely the responsibility of the authors and do not reflect the views of the NHMRC. 


\section{Declarations}

Publication of this article was funded by the National Health and Medical Research Council Centre for Research Excellence in Advanced Cardio-respiratory Therapies Improving OrgaN Support (ACTIONS).

\section{Published online: 16 March 2016}

\section{References}

1. Beurtheret $\mathrm{S}$, Mordant $\mathrm{P}$, Paoletti $\mathrm{X}$, et al. Emergency circulatory support in refractory cardiogenic shock patients in remote institutions: a pilot study (the cardiac-RESCUE program). Eur Heart J. 2013;34:112-20.

2. Reynolds HR, Hochman JS. Cardiogenic shock: current concepts and improving outcomes. Circulation. 2008;117:686-97.

3. Shekar K, Mullany DV, Thomson B, Ziegenfuss M, Platts DG, Fraser JF. Extracorporeal life support devices and strategies for management of acute cardiorespiratory failure in adult patients: a comprehensive review. Crit Care. 2014;18:219.

4. Abrams D, Combes A, Brodie D. Extracorporeal membrane oxygenation in cardiopulmonary disease in adults. J Am Coll Cardiol. 2014;63:2769-78.

5. Stub D, Bernard S, Pellegrino V, et al. Refractory cardiac arrest treated with mechanical CPR, hypothermia, ECMO and early reperfusion (the CHEER trial). Resuscitation. 2015:86:88-94.

6. Pinney SP. Left ventricular assist devices: The adolescence of a disruptive technology. J Card Fail. 2015;21:824-34.

7. Cohn WE, Timms DL, Frazier OH. Total artificial hearts: past, present, and future. Nat Rev Cardiol. 2015;12:609-17.

8. Thiele H, Zeymer U, Neumann FJ, et al. Intraaortic balloon support for myocardial infarction with cardiogenic shock. N Engl J Med. 2012;367:1287-96

9. Cove ME, MacLaren G. Clinical review: mechanical circulatory support for cardiogenic shock complicating acute myocardial infarction. Crit Care. 2010;14:235

10. Combes A, Leprince P, Luyt CE, et al. Outcomes and long-term quality-oflife of patients supported by extracorporeal membrane oxygenation for refractory cardiogenic shock. Crit Care Med. 2008:36:1404-11.

11. Extracorporeal Life Support Organization. ECLS Registry Report International Summary. 2015. https://www.elso.org/Registry/Statistics/ InternationalSummary.aspx. Accessed Nov 2015

12. ELSO. Guidelines for Adult Cardiac Failure Supplement Version 1.3. 2013. https://www.elso.org/Portals/O/IGD/Archive/FileManager/e76ef78 eabcusersshyerdocumentselsoguidelinesforadultcardiacfailure1.3.pdf. Accessed Nov 2015

13. Chen YS, Lin JW, Yu HY, et al. Cardiopulmonary resuscitation with assisted extracorporeal life-support versus conventional cardiopulmonary resuscitation in adults with in-hospital cardiac arrest: an observational study and propensity analysis. Lancet. 2008;372:554-61.

14. Thiagarajan RR. Extracorporeal membrane oxygenation to support cardiopulmonary resuscitation: Useful, but for whom? Crit Care Med. 2011;39:190-1.

15. Feldman D, Pamboukian SV, Teuteberg JJ, et al. The 2013 International Society for Heart and Lung Transplantation Guidelines for mechanical circulatory support: Executive summary. J Heart Lung Transplant. 2013;32:157-87.

16. Combes A, Bacchetta M, Brodie D, Muller T, Pellegrino V. Extracorporeal membrane oxygenation for respiratory failure in adults. Curr Opin Crit Care. 2012;18:99-104.

17. Aiyagari RM, Rocchini AP, Remenapp RT, Graziano JN. Decompression of the left atrium during extracorporeal membrane oxygenation using a transseptal cannula incorporated into the circuit. Crit Care Med. 2006;34:2603-066.

18. Guirgis M, Kumar K, Menkis AH, Freed DH. Minimally invasive left-heart decompression during venoarterial extracorporeal membrane oxygenation: an alternative to a percutaneous approach. Interact Cardiovasc Thorac Surg. 2010;10:672-4

19. Vlasselaers D, Desmet M, Desmet L, Meyns B, Dens J. Ventricular unloading with a miniature axial flow pump in combination with extracorporeal membrane oxygenation. Intensive Care Med. 2006;32:3293.

20. Aggarwal A, Modi S, Kumar S, et al. Use of a single-circuit CentriMag(R) for biventricular support in postpartum cardiomyopathy. Perfusion. 2013;28:156-9.

21. Massetti M, Gaudino M, Saplacan V, Farina P. From extracorporeal membrane oxygenation to ventricular assist device oxygenation without sternotomy. J Heart Lung Transplant. 2013;32:138-9.
22. Prutkin JM, Strote JA, Stout KK. Percutaneous right ventricular assist device as support for cardiogenic shock due to right ventricular infarction. J Invasive Cardiol. 2008;20:E215-6.

23. Rajagopal V, Steahr G, Wilmer Cl, Raval NY. A novel percutaneous mechanical biventricular bridge to recovery in severe cardiac allograft rejection. J Heart Lung Transplant. 2010;29:93-5.

24. Garbade J, Bittner HB, Barten MJ, Mohr FW. Current trends in implantable left ventricular assist devices. Cardiol Res Pract. 2011;2011:290561.

25. Stewart GC, Givertz MM. Mechanical circulatory support for advanced heart failure: patients and technology in evolution. Circulation. 2012;125:1304-15.

26. Drakos SG, Janicki L, Horne BD, et al. Risk factors predictive of right ventricular failure after left ventricular assist device implantation. Am J Cardiol. 2010;105:1030-5

27. Cowger J, Sundareswaran K, Rogers JG, et al. Predicting survival in patients receiving continuous flow left ventricular assist devices: the HeartMate II risk score. J Am Coll Cardiol. 2013;61:313-21.

28. Teuteberg JJ, Ewald GA, Adamson RM, et al. Risk assessment for continuous flow left ventricular assist devices: does the destination therapy risk score work? An analysis of over 1,000 patients. J Am Coll Cardiol. 2012;60:44-51.

29. Kirklin JK, Naftel DC, Pagani FD, et al. Sixth INTERMACS annual report: A 10,000-patient database. J Heart Lung Transplant. 2014;33:555-64.

30. Rose EA, Gelijns AC, Moskowitz AJ, et al. Long-term use of a left ventricular assist device for end-stage heart failure. N Engl J Med. 2001;345:1435-43.

31. Slaughter MS, Rogers JG, Milano CA, et al. Advanced heart failure treated with continuous-flow left ventricular assist device. N Engl J Med. 2009;361:2241-51.

32. Sundareswaran KS, Reichenbach SH, Masterson KB, Butler KC, Farrar DJ. Low bearing wear in explanted HeartMate II left ventricular assist devices after chronic clinical support. ASAIO J. 2013;59:41-5.

33. Thoratec Corporation. HeartMate II Clinical Outcomes. http://www.thoratec. com/vad-trials-outcomes/clinical-outcomes/hm2-ce-phase1.aspx. Accessed November 2015

34. Timms D. A review of clinical ventricular assist devices. Med Eng Phys. 2011;33:1041-7.

35. Krabatsch T, Potapov E, Stepanenko A, et al. Biventricular circulatory support with two miniaturized implantable assist devices. Circulation. 2011;124:179-86.

36. Slaughter MS, Sobieski MA, Tamez D, et al. HeartWare miniature axial-flow ventricular assist device: design and initial feasibility test. Texas Heart Inst J. 2009;36:12-6.

37. Fitzpatrick JR, Frederick JR, Hiesinger W, et al. Early planned institution of biventricular mechanical circulatory support results in improved outcomes compared with delayed conversion of a left ventricular assist device to a biventricular assist device. J Thorac Cardiovasc Surg. 2009;137:971-7.

38. Milano CA, Simeone AA. Mechanical circulatory support: devices, outcomes and complications. Heart Fail Rev. 2013;18:35-53.

39. Frazier $\mathrm{OH}$, Cohn WE. Continuous-flow total heart replacement device implanted in a 55-year-old man with end-stage heart failure and severe amyloidosis. Texas Heart Inst J. 2012;39:542-6.

40. Pirk J, Maly J, Szarszoi O, et al. Total artificial heart support with two continuous-flow ventricular assist devices in a patient with an infiltrating cardiac sarcoma. ASAIO J. 2013:59:178-80.

41. Strueber M, Schmitto JD, Kutschka I, Haverich A. Placement of 2 implantable centrifugal pumps to serve as a total artificial heart after cardiectomy. J Thorac Cardiovasc Surg. 2012;143:507-9.

42. SynCardia Systems Inc. Total Artificial Heart Facts. http://www.syncardia. com/total-facts/total-artificial-heart-facts.html. Accessed November 2015

43. Abrams DC, Prager K, Blinderman CD, Burkart KM, Brodie D. Ethical dilemmas encountered with the use of extracorporeal membrane oxygenation in adults. Chest. 2014;145:876-82.

44. Rizzieri AG, Verheijde JL, Rady MY, McGregor JL. Ethical challenges with the left ventricular assist device as a destination therapy. Philos Ethics Humanit Med. 2008:3:20.

45. Bruce CR. A review of ethical considerations for ventricular assist device placement in older adults. Aging Dis. 2013:4:100-12.

46. Kar B, Basra SS, Shah NR, Loyalka P. Percutaneous circulatory support in cardiogenic shock: interventional bridge to recovery. Circulation. 2012;125:1809-17.

47. Uriel N, Pak SW, Jorde UP, et al. Acquired von Willebrand syndrome after continuous-flow mechanical device support contributes to a high prevalence of bleeding during long-term support and at the time of transplantation. J Am Coll Cardiol. 2010;56:1207-13. 
48. Geisen U, Heilmann C, Beyersdorf F, et al. Non-surgical bleeding in patients with ventricular assist devices could be explained by acquired von Willebrand disease. Eur J Cardiothorac Surg. 2008;33:679-84.

49. Cheng A, Williamitis CA, Slaughter MS. Comparison of continuous-flow and pulsatile-flow left ventricular assist devices: is there an advantage to pulsatility? Ann Cardiothorac Surg. 2014;3:573-81.

50. Holman WL, Kirklin JK, Naftel DC, et al. Infection after implantation of pulsatile mechanical circulatory support devices. J Thorac Cardiovasc Surg. 2010;139:1632-6.

51. Aissaoui N, Morshuis $M$, Schoenbrodt $M$, et al. Temporary right ventricular mechanical circulatory support for the management of right ventricular failure in critically ill patients. J Thorac Cardiovasc Surg. 2013;146:186-91.

52. Salamonsen RF, Mason DG, Ayre PJ. Response of rotary blood pumps to changes in preload and afterload at a fixed speed setting are unphysiological when compared with the natural heart. Artif Organs. 2011;35:E47-53.

53. Arroyo D, Cook S. Percutaneous ventricular assist devices: new deus ex machina? Minim Invasive Surg. 2011;2011:604397.

54. Bourque K, Gernes DB, Loree 2nd HM. HeartMate III: pump design for a centrifugal LVAD with a magnetically levitated rotor. ASAIO J. 2001;47:401-5. 